\title{
Cerebral Malaria with Corpus Callosum Splenium Lesion
}

\author{
Amber Eker' ${ }^{(0)}$, H. Kaya Süer ${ }^{2}$ (i), Özgür Tosun ${ }^{3}$ (i) \\ 'Department of Neurology, Near East University School of Medicine, Nicosia, Cyprus \\ 2Department of Clinical Microbiology and Infectious Diseases, Near East University School of Medicine, Nicosia, Cyprus \\ ${ }^{3}$ Department of Radiology, Katip Çelebi University School of Medicine, İzmir, Turkey
}

ORCID IDs of the authors: A.E. 0000-000I-9997-4662; H.K.S. 0000-0002-2565-3425; Ö.T. 0000-000I-6755-2I3I.

Cite this article as: Eker A, Tosun Ö, Süer HK. Cerebral Malaria with Corpus Callosum Splenium Lesion. Cyprus J Med Sci 2019; 4(2): 146-50.

\begin{abstract}
Malaria is still an important public health problem especially in less developed and developing countries. Cerebral malaria is one of the most severe complications of malaria. Mortality is high, and some neurocognitive problems may be observed in surviving patients during the long-term follow-up. A 27-year-old man was admitted to the emergency department with fever, vomiting, diarrhea, muscle pain, fatigue, headache, progressive drowsiness, and seizure. His cranial magnetic resonance imaging revealed focal T2 hyperintensity in the corpus callosum splenium. His blood smears showed diagnostic crescent-shaped gametocytes for Plasmodium falciparum malaria. His physical and neurological examinations were normal at discharge. Corpus callosum splenium involvement is really rare, but if observed, it is suggestive for diagnosis in patients with suspected cerebral malaria. Malaria is not only a problem in endemic areas, but it is a problem worldwide in this globalization age. We all have to be familiar to the systemic and also central nervous system complications of malaria.
\end{abstract}

Keywords: Malaria, cerebral malaria, corpus callosum splenium

\section{INTRODUCTION}

Annually, over 500 million clinical malaria cases have been observed worldwide, thereby causing over I million deaths. Cerebral malaria is one of the most severe complications of infection with Plasmodium falciparum malaria. Mortality is high, and some neurocognitive problems may be observed in surviving patients during the long-term follow-up. Cerebral malaria is seen in $2 \%$ of malaria cases. It mainly affects children in endemic areas. Cerebral malaria frequency is rarer in adults than in children $(1,2)$.

Cerebral malaria may cause diffuse involvement in the brain; therefore, wide spectrum neurological manifestations can be observed. Altered consciousness and coma are the most common neurological manifestations, followed by seizures. In addition to transient extrapyramidal, neuropsychiatric manifestations, and focal motor, sensorial signs may occur. Approximately one-third of patients with cerebral malaria die $(2,3)$.

Cranial magnetic resonance imaging (MRI) can be normal despite the neurological signs. However, various reports of MRI in cerebral malaria have revealed focal or diffuse signal changes in the centrum semiovale, corpus callosum, basal ganglia, cortex, cerebellum, and brainstem (2). Here we report a case of a well-treated adult patient with cerebral malaria who has a corpus callosum splenium lesion.

\section{CASE PRESENTATION}

A 27-year-old man was admitted to our emergency department with fever, vomiting, diarrhea, muscle pain, headache, and progressive drowsiness. The patient is originally from Turkey and studying in Cyprus for 2 years. He had regular work-related travel history to Sierra Leone. He had never used any prophylactic medicine for malaria. On physical examination, tachycardia, icterus, hepatosplenomegaly, and anemia were found. His body temperature was $38.3^{\circ} \mathrm{C}$. On neurological examination, he was lethargic and had a Glasgow Coma Scale score of 6. He had meningeal irritation signs with neck stiffness. Additionally, convulsions with nystagmus, generalized tonic spasms, and apnea were observed. Cranial MRI was performed urgently and revealed focal T2 hyperintensity in the corpus callosum splenium that showed diffusion restriction in diffusion-weighted imaging $(D W I)$ and apparent diffusion coefficient $(A D C)$. Additionally, any contrast enhancement in the parenchyma and meninges 
was not observed (Figure I). On electroencephalography (EEG), generalized severe background slowing was found (Figure 2a).

His laboratory results were as follows: hemoglobin $8.7 \mathrm{~g} / \mathrm{dL}$, white blood cells $16,000 / \mathrm{mL}$, platelets $59,000 / \mathrm{mL}$, erythrocyte sedimentation rate $65 \mathrm{~mm} / \mathrm{h}$, C-reactive protein $20 \mathrm{mg} / \mathrm{dL}$, aspartate aminotransferase $80 \mathrm{U} / \mathrm{L}$, alanine aminotransferase $149 \mathrm{U} / \mathrm{L}$, lactate dehydrogenase $1640 \mathrm{U} / \mathrm{L}$, gamma-glutamyl transferase $88 \mathrm{U} / \mathrm{L}$, total bilirubin $5.64 \mathrm{mg} / \mathrm{dL}$, indirect bilirubin $5.07 \mathrm{mg} / \mathrm{dL}$, glucose $108 \mathrm{mg} / \mathrm{dL}$, urea $49 \mathrm{mg} / \mathrm{dL}$, creatinine $1.03 \mathrm{mg} / \mathrm{dL}$, sodium 136 $\mathrm{mmol} / \mathrm{L}$, and potassium $3.2 \mathrm{mmol} / \mathrm{L}$. Hepatitis markers for hepatitis $B$ and $C$ and human immunodeficiency virus infections were all negative. On urine examination, a dark, hematuric urine with many erythrocytes was found. On abdominal ultrasonography, hepatosplenomegaly and focal spleen infarction were reported.
His blood smears showed acanthocytosis, ring-shaped trophozoites, and schizonts in erythrocytes, ruptured schizonts, and crescent-shaped gametocytes that resemble P. falciparum infection (Figure 3).

Antimalarial treatments with intravenous artemether and lumefantrine, quinine, and tetracycline tablets were started, and the patient regained consciousness within 3 days. Antiepileptic medication with levetiracetam was also started. Control EEG was performed on week I and showed normal background activity (Figure 2b).

All his neurological examination and detailed neurocognitive evaluation were normal at discharge. Consent was obtained from the patient to report the features of this case.
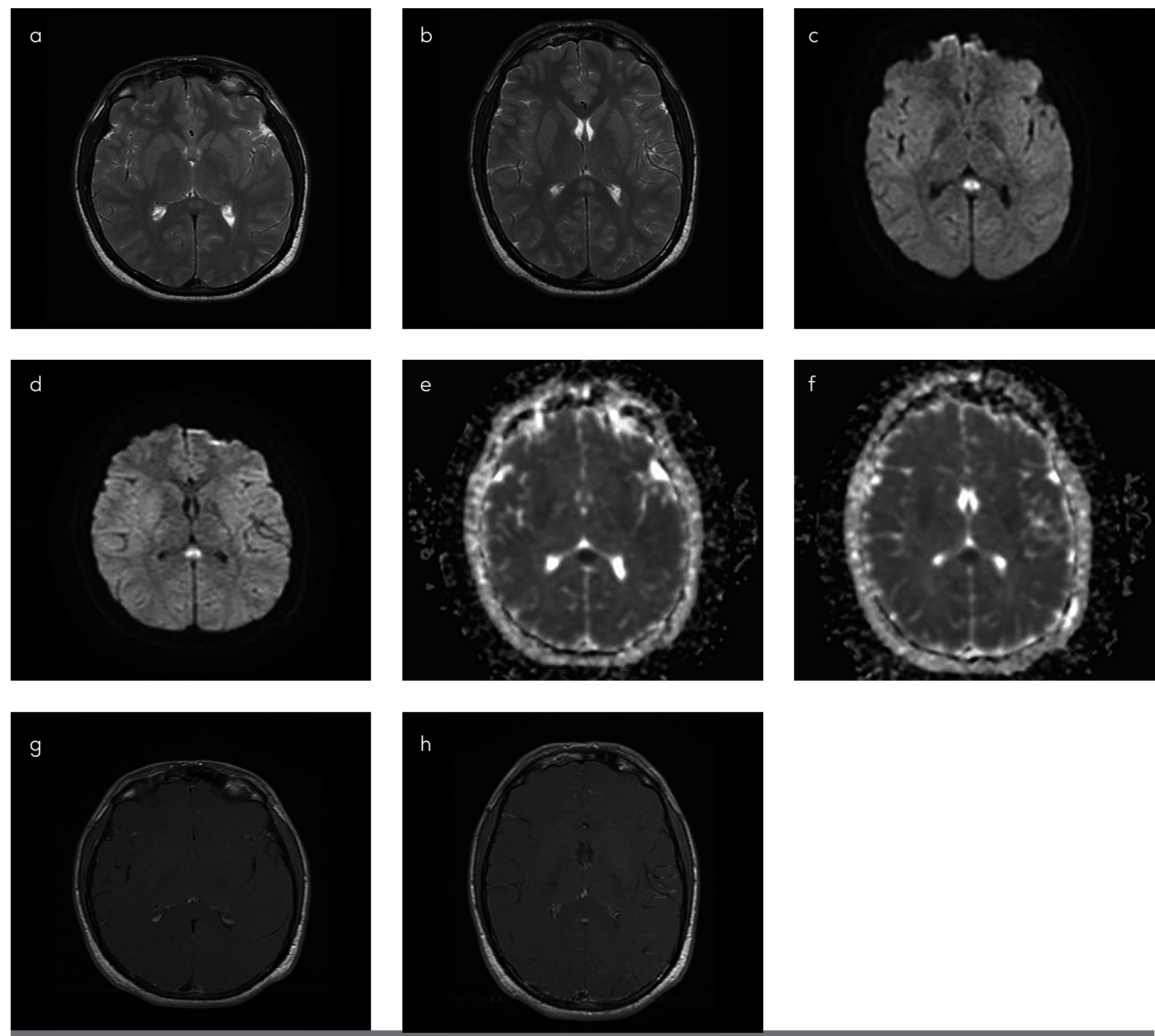

FIGURE I. a-h. Cerebral MRI at admission showing a lesion of the splenium of corpus callosum with high signal intensity on T2-weighted sequence $(a, b)$, DWI (c, d), and low signal intensity on ADC map images (e-f). Lesion does not reveal contrast enhancement on TI-weighted sequence $(g, h)$ 

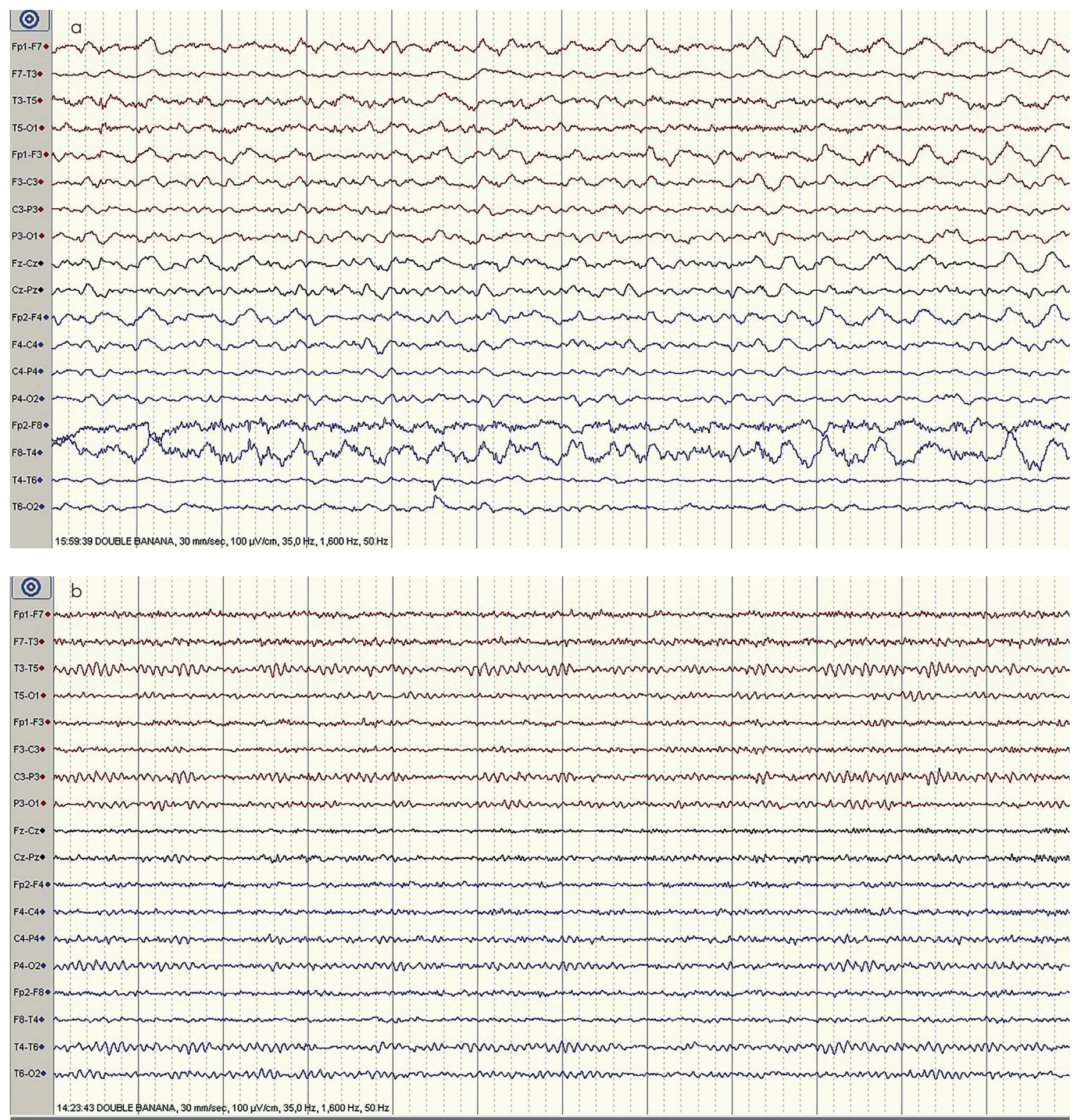

FIGURE 2. a, b. Initial EEG shows generalized severe background slowing with $3 \mathrm{~Hz}$ delta waves (a). Control EEG shows normal background activity (b)

\section{DISCUSSION}

Cerebral malaria is one of the most severe complications of infection with $P$. falciparum malaria. The pathogenesis of cerebral malaria has been explained by two mechanisms: vascular sequestration of parasitized erythrocytes and potential cerebral toxicity by cytokines. Parasitized red blood cell sequestration in brain small capillaries results in impaired perfusion and local release of inflammatory factors. Released cytokines trigger vasodilatation, cerebral edema, and ischemia (I-4).
This pathomechanism results in cerebral swelling, intracranial hypertension, brainstem involvement, arterial-venous infarction, and retinal changes or papilledema.

The widespread involvement in the central nervous system causes a wide spectrum of clinical findings, such as confusion, seizures, and problems in posture, ocular movements, and respiratory patterns. The clinical hallmark of cerebral malaria is impaired consciousness that may progress to coma. Our patient also had coma with the systemic involvement of malaria. After 

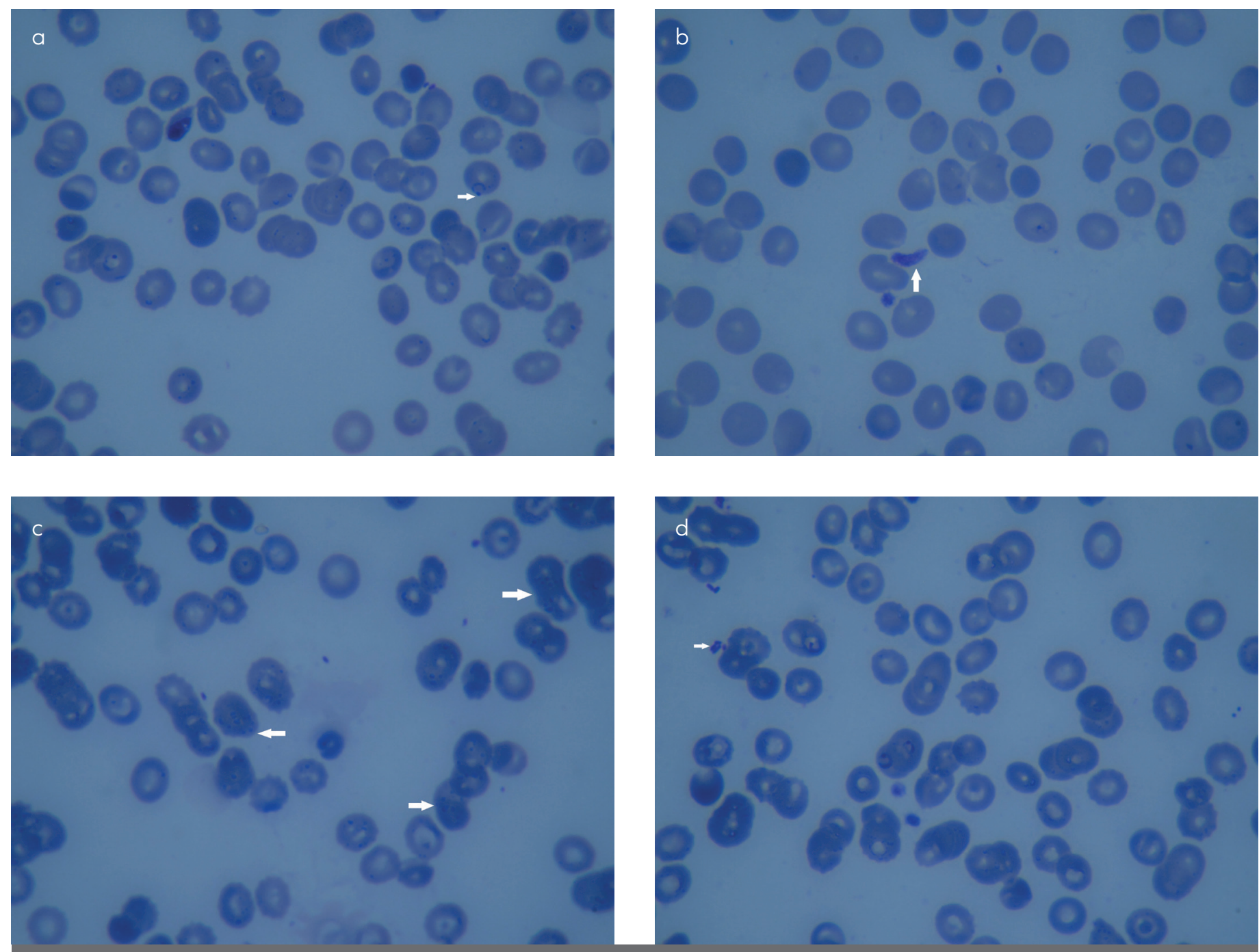

FIGURE 3. a-d. Blood smear shows acanthocytosis, ring-shaped trophozoites (a), schizonts in erythrocytes (c), ruptured schizonts (d), and crescent-shaped gametocytes (b)

exclusion of all possible other reasons for coma, this patient was diagnosed with cerebral malaria.

Cranial MRI can be normal despite the neurological signs. However, various reports of MRI in cerebral malaria have revealed focal or diffuse signal changes in the centrum semiovale, corpus callosum, basal ganglia, thalamus, cortex, and brainstem. Pontin and upper medulla myelinolysis and cerebellar demyelination have also been reported. Microinfarcts or microbleedings of the cerebral hemispheres, brainstem, cerebellum, and venous thrombosis or infarcts can be observed. The hyperintensities on T2-weighted images were considered to be due to edema, ischemia, toxic injury, or gliosis. DWI changes usually do not occur in a vascular distribution because of local ischemia due to a high metabolic demand, resulting from reduced blood flow, focal hypoglycemia, seizures, or hypoxia (2-5).

The largest published prospective imaging study on cerebral malaria includes 120 Malawian children. The researchers summarized all the MRI features of cerebral malaria. T2 signal changes in the corpus callosum were reported in nearly half of the study population. They also observed that corpus callosum involvement was associated with positive DWI changes, and some cases had predominantly diffuse involvement of the splenium. They report that corpus callosum DWI abnormalities were observed in 6 of 32 cases without retinopathy and in 52 of 120 with retinopathy. Additionally, 5 in 6 of retinopathy negative cases and 38 in 52 of retinopathy positive cases with corpus callosum involvement had splenium predominance (4).

These studies also reported corpus callosum involvement without any diffusion restriction in DWI. Distinctively, our case had acute splenium diffusion restriction in MRI in the early period of his disease. Cytotoxic edema via excitotoxic and inflammatory injury is most likely the main mechanism in corpus callosum splenium involvement in malaria. Fever, seizures, and ischemic injury due to vascular sequestration of infected erythrocytes are the other discussed mechanisms $(2-4,6,7)$.

The transient lesions of the corpus callosum splenium have also been described in patients with viral infections, antiepileptic drug toxicity or withdrawal, high-altitude cerebral edema, metabolic disorders, Wernicke encephalopathy, MarchiafavaBignami disease, hemolytic uremic syndrome, and traumatic axonal injury. The researchers hypothesize that these reversible lesions are due to intramyelinic edema in this area $(2,4,6,7)$. 
Corpus callosum splenium involvement is rare, but if observed, it may be suggestive for diagnosis in patients with suspected cerebral malaria. Cerebral malaria should be considered in the list of possible causes of reversible lesion of the corpus callosum splenium. Our knowledge regarding adult cerebral malaria is very limited. Increasing case reports help to improve our knowledge with regard to imaging findings in adult cerebral malaria.

Malaria is not only a problem in endemic areas, but it is a problem globally in this globalization age. We all have to be familiar to the systemic and also central nervous system complications of malaria. Early diagnosis and effective treatment prevent radiological sequel, early mortality, and long-term cognitive dysfunction.

Informed Consent: Written informed consent was obtained from the patient who participated in this study.

Peer-review: Externally peer-reviewed.

Author contributions: Concept - A.E., H.K.S, O.T.; Design - A.E., H.K.S., O.T.; Supervision - A.E., H.K.S., O.T; Resource - A.E., H.K.S., O.T.; Materials - A.E., H.K.S., O.T.; Data Collection and/or Processing - A.E., H.K.S., O.T.; Analysis and/or Interpretation - A.E., H.K.S., O.T.; Literature Search - A.E., H.K.S., O.T.; Writing - A.E., H.K.S., O.T.; Critical Reviews - A.E., H.K.S., O.T.
Conflict of Interest: The authors have no conflicts of interest to declare.

Financial Disclosure: The authors declared that this study has received no financial support.

\section{REFERENCES}

I. Idro R, Marsh K, John CC, Newton CR. Cerebral malaria: mechanisms of brain injury and strategies for improved neurocognitive outcome. Pediatr Res 2010; 68: 267-74. [CrossRef]

2. Rasalkar DD, Paunipagar BK, Sanghvi D, Sonawane BD, Loniker P. Magnetic resonance imaging in cerebral malaria: a report of four cases. Br J Radiol 20II; 84: 380-5. [CrossRef]

3. Cordoliani YS, Sarrazin JL, Felten D, Caumes E, Lévêque C, Fisch A. MR of cerebral malaria. AJNR Am J Neuroradiol 1998; 19: 8714.

4. Potchen MJ, Kampondeni SD, Seydel KB, Birbeck GL, Hammond CA, Bradley WG, et al. Acute brain MRI findings in 120 Malawian children with cerebral malaria: new insights into an ancient disease. AJNR Am J Neuroradiol 2012; 33: 1740-6. [CrossRef]

5. Yadav P, Sharma R, Kumar S, Kumar U. Magnetic resonance features of cerebral malaria. Acta Radiol 2008; 49: 566-9. [CrossRef]

6. Garcia-Monco JC, Cortina IE, Ferreira E, Martínez A, Ruiz L, Cabrera A, et al. Reversible splenial lesion syndrome (RESLES): what's in a name? J Neuroimaging 20II; 2l: el-4. [CrossRef]

7. Hantson P, Hernalsteen D, Cosnard G. Reversible splenial lesion syndrome in cerebral malaria. J Neuroradiol 20I0; 37: 243-6. [CrossRef] 